\title{
ON THE KUMMER SURFACE
}

\author{
BY W. V. PARKER
}

1. Introduction.* In his paper On hyperelliptic functions of genus two, A. L. Dixon $\dagger$ chooses as fundamental the curve, $H$, whose equation in the $(r, s)$ plane may be written in the form

$$
s^{2}=f(r) \equiv r^{5}+a r^{4}+b r^{3}+c r^{2}+r .
$$

The Kummer surface associated with this curve presents several interesting features which are not found in the more usual forms. Its equation is quadratic in two of the variables. By introducing homogeneous coordinates we find that its equation possesses a three way symmetry not found in the other forms. In this form the surface has two vertices of the tetrahedron of reference as nodes and two of its faces as tropes, whereas in the usual forms we have only one of these. $\ddagger$

2. The Associated Kummer Surface. Consider the hyperelliptic curve $H$ of genus two in the $(r, s)$ plane, whose equation is

$$
s^{2}=r^{5}+a r^{4}+b r^{3}+c r^{2}+r,
$$

where $a, b, c$ are real and subject only to the condition that the right member does not have multiple zeros. Denote the branch points of the Riemann surface of $H$ by $0, e_{1}, e_{2}, e_{3}, e_{4}, \infty$.

Let $\left(\alpha_{1}, \rho_{1}\right)$ and $\left(\alpha_{2}, \rho_{2}\right)$ be any two points on $H$, not necessarily on the real part of $H$. If we write

$$
\begin{aligned}
2 F\left(\alpha_{1}, \alpha_{2}\right) & \equiv \alpha_{1}^{2} \alpha_{2}^{2}\left(\alpha_{1}+\alpha_{2}\right) \\
& +2 a \alpha_{1}^{2} \alpha_{2}^{2}+b \alpha_{1} \alpha_{2}\left(\alpha_{1}+\alpha_{2}\right)+2 c \alpha_{1} \alpha_{2}+\alpha_{1}+\alpha_{2},
\end{aligned}
$$

equation (1) becomes $s^{2}=F(r, r)$. Let us now introduce homogeneous variables $(x, y, z, t)$ symmetrically related to these two points by the formulas

* This paper is a part of a dissertation written in Brown University, 1931. The author is indebted to Professor A. A. Bennett for many helpful suggestions.

$\dagger$ Quarterly Journal of Mathematics, vol. 36 (1904), p. 1.

$\ddagger$ Baker, Multiply Periodic Functions, Cambridge University Press, 1907, Chapter 3. 
(3) $\frac{x}{t}=\alpha_{1}+\alpha_{2}, \quad \frac{y}{t}=\alpha_{1} \alpha_{2}, \quad \frac{z}{t}=\frac{2 F\left(\alpha_{1}, \alpha_{2}\right)-2 \rho_{1} \rho_{2}}{\left(\alpha_{1}-\alpha_{2}\right)^{2}}$.

If now we eliminate $\alpha_{1}$ and $\alpha_{2}$ and incidentally $\rho_{1}$ and $\rho_{2}$ from equations (3), we find that $x, y, z, t$ are connected by the relation

$$
Y^{2}-4 X Z=0
$$

where

(5) $X=a y t+x y+z t, Y=b y t-y^{2}-t^{2}-x z, Z=c y t+y z+x t$.

The relation (4) is the equation of a Kummer surface $K$ associated with $H$. The form of (4) is unaltered if we interchange $x$ with $z$ and $y$ with $t, a$ with $c$ and $x$ with $z$, or $a$ with $c$ and $y$ with $t$.

To any two points of $H$ there corresponds a single point of $K$. A point of $K$ corresponds to two pairs of points on $H$ which are obtained the one from the other by reflection in the $r$-axis. There is not, therefore, a one-to-one reciprocal correspondence between the points of $K$ and the pairs of points of $H$. Such a correspondence may be established, however, by considering $K$ as a kind of double spread. In order to distinguish between the points of the two spreads, we may introduce another pair of functions such as

$$
\eta=\frac{\alpha_{2} \rho_{1}-\alpha_{1} \rho_{2}}{\alpha_{1} \alpha_{2}\left(\alpha_{1}-\alpha_{2}\right)}, \quad \zeta=\frac{\alpha_{1}^{2} \rho_{2}-\alpha_{2}^{2} \rho_{1}}{\alpha_{1} \alpha_{2}\left(\alpha_{1}-\alpha_{2}\right)} .
$$

These functions satisfy the relations

$$
y t \eta^{2}=X, 2 y t \eta \zeta=Y, y t \zeta^{2}=Z,
$$

where $X, Y, Z$ are as defined by (5).

All points of $K$ which correspond to a fixed value $\alpha_{1}$ of $r$ together with any other value $\alpha_{i}$ lie in the plane

$$
\alpha_{\ddagger} x-y-\alpha_{1}^{2} t=0 \text {. }
$$

For if we let $t=1$, we have $x=\alpha_{1}+\alpha_{i}, y=\alpha_{1} \alpha_{i}$, which substituted in (7) gives $\alpha_{1}\left(\alpha_{1}+\alpha_{i}\right)-\alpha_{1} \alpha_{i}-\alpha_{1}^{2}=0$, an identity in $\alpha_{i}$. The two points of $K$ corresponding to a given pair of values $\alpha_{1}$ and $\alpha_{2}$ of $r$ are, therefore, in both the planes $\alpha_{1} x-y-\alpha_{1}^{2} t=0$ and 
$\alpha_{2} x-y-\alpha_{2}{ }^{2} t=0$ and hence on a line through $(0,0,1,0)$. In case $\alpha_{1}$ or $\alpha_{2}$ is a branch point these two points of $K$ coincide and the line from this point to $(0,0,1,0)$ is tangent to $K$ at the point, for, as will be shown presently, if $\alpha_{1}$ is a branch point the plane (7) is a trope.

If both $\alpha_{1}$ and $\alpha_{2}$ are branch points, it is readily shown that the corresponding point of $K$ is a node and the plane

$$
\alpha_{1} \alpha_{2} x-\left(\alpha_{1}+\alpha_{2}\right) y+z-2\left(\alpha_{1}-\alpha_{2}\right)^{-2} F\left(\alpha_{1}, \alpha_{2}\right) t=0
$$

is a trope ${ }^{*}$ that is, a plane tangent to the surface along a conic. In particular if $\alpha_{1}=\alpha_{2}$ the node is $(0,0,1,0)$ and the trope is $t=0$, and if $\alpha_{1}=0, \alpha_{2}=\infty$ the node is $(1,0,0,0)$ and the trope is $y=0$.

Since both $\rho_{1}$ and $\rho_{2}$ are zero when $\alpha_{1}$ and $\alpha_{2}$ are finite branch points, we see that if the sixteen nodes are $\left(x_{i}, y_{i}, z_{i}, t_{i}\right),(i=1$, $2, \cdots, 16)$, the equations of the sixteen tropes are

$$
\left|\begin{array}{cc}
x_{i} & y_{i} \\
x & y
\end{array}\right|+\left|\begin{array}{cc}
z_{i} & t_{i} \\
z & t
\end{array}\right|=0 .
$$

We establish in this way a one-to-one reciprocal correspondence between the nodes and tropes. There are, however, six nodes on each trope and six tropes on each node. We may determine how these are situated from the following table. Let the node (or trope) obtained by taking 0 with $\infty$ be denoted by (0), that obtained by taking 0 with $e_{i}$ by $(i)$, that obtained by taking $e_{i}$ with $e_{j}$ by $(i, j)$, that obtained by taking $\infty$ with $e_{l}$ by $(i, j, k)$ and that obtained by taking any branch point with itself by $(1,2,3,4)$, where $i, j, k, l$ denote the numbers $1,2,3,4$ in some order. If the symbol outside denotes the node the ones inside denote the tropes on it, and if the symbol outside de-

\begin{tabular}{|c|c|c|c|c|c|}
\hline$(0)[(0)$ & (1) & (2) & (3) & (4) & $(1,2,3,4)]$ \\
\hline$(i)[(0)$ & (i) & $(j, k)$ & $(k, l)$ & $(l, j)$ & $(j, k, l)$ \\
\hline$(i, j)[(k)$ & $(l)$ & $(i, j)$ & $(k, l)$ & $(i, k, l)$ & $(j, k, l)$ \\
\hline$(i, j, k)[(l)$ & $(i, l)$ & $(j, l)$ & $(k, l)$ & $(i, j, k)$ & $(1,2,3,4)]$ \\
\hline$(1,2,3,4)[(0)$ & $(1,2,3)$ & $(2,3,4)$ & $(3,4,1)$ & $(4,1,2)$ & $(1,2,3,4)]$ \\
\hline
\end{tabular}
notes the trope the ones inside denote the nodes on it.

* Baker, loc. cit., Chapter 3. 
Equation (4) of the surface is quadratic in the variables $x$ and $z$. If we write it in expanded form as a quadratic in $z$ we have

$$
\begin{aligned}
\left(x^{2}\right. & -4 y t) z^{2}-2\left(x y^{2}+b x y t+2 a y^{2} t+x t^{2}+2 c y t^{2}\right) z+y^{4} \\
& -2 b y^{3} t-4 c x y^{2} t-4 x^{2} y t+\left(2+b^{2}-4 a c\right) y^{2} t^{2} \\
& -4 a x y t^{2}-2 b y t^{3}+t^{4}=0 .
\end{aligned}
$$

The discriminant of this quadratic in $z$ may be written $\Delta_{z}=y t\left(e_{1} x-y-e_{1}^{2} t\right)\left(e_{2} x-y-e_{2}^{2} t\right)\left(e_{3} x-y-e_{3}^{2} t\right)\left(e_{4} x-y-e_{4}^{2} t\right)$.

Each of these linear factors equated to zero is the equation of a trope on the node $(0,0,1,0)$. A similar thing is true for the discriminant if we consider the equation as a quadratic in $x$. In this case we get the tropes on the node $(1,0,0,0)$.

3. A Numerical Example. The correspondence between pairs of points of $H$ and the real points of $K$ is not as simple as we might expect. To add to the clarity of the general discussion with particular reference to reality, let us consider a particular Kummer surface of the type just discussed. Many of the facts found here are true for the general case when $e_{1}, e_{2}, e_{3}, e_{4}$ are all real.

Take as the fundamental curve $H$,

$$
s^{2}=r^{5}-\frac{3}{2} r^{4}-2 r^{3}+\frac{3}{2} r^{2}+r
$$

for which $e_{1}=1, e_{2}=-1, e_{3}=2, e_{4}=-\frac{1}{2}$. This choice of the subscripts for the $e$ 's is absolutely arbitrary. The equation of the corresponding Kummer surface for this curve is

(2) $y^{4}-2 x y^{3} z+x^{2} z^{2}+\left(4 y^{3}+4 x y z-6 x y^{2}-4 x^{2} y+6 y^{2} z-4 y z^{2}\right) t$

$$
+\left(15 y^{2}-2 x z-6 y z+6 x y\right) t^{2}+4 y t^{3}+t^{4}=0 \text {. }
$$

We get real points on the surface in the following cases:

1. $\alpha_{1}$ and $\alpha_{2}$ real with $\rho_{1}$ and $\rho_{2}$ real.

2. $\alpha_{1}$ and $\alpha_{2}$ real with $\rho_{1}$ and $\rho_{2}$ pure imaginary numbers.

3. $\alpha_{1}$ and $\alpha_{2}$ conjugate complex numbers with $\rho_{1}$ and $\rho_{2}$ conjugate complex numbers.

4. $\alpha_{1}$ and $\alpha_{2}$ conjugate complex numbers with $\rho_{2}$ equal to the negative of the conjugate of $\rho_{1}$. 
In order for $\alpha_{1}$ and $\alpha_{2}$ to change continuously from both real to conjugate complex numbers they must become equal. Points for which $\alpha_{1}=\alpha_{2}$ lie on the cylinder

$$
x^{2}-4 y t=0 .
$$

This cylinder separates the real points of the surface for which $\alpha_{1}$ and $\alpha_{2}$ are real from those for which $\alpha_{1}$ and $\alpha_{2}$ are conjugate complex numbers. Points on the real part of the surface outside of the cylinder correspond to real values of $\alpha_{1}$ and $\alpha_{2}$, while those inside correspond to conjugate complex values of $\alpha_{1}$ and $\alpha_{2}$.

The surfaces consists of eight pieces, two entirely finite and six extending to infinity. Each piece joins with four others, one at each of its nodes. Each infinite piece has two of its nodes at infinity. Two of the infinite pieces are cut by the cylinder (3) and each of these pieces is divided into six portions by it, three lying outside and three inside.

The real values of $r$ are divided into six intervals by the branch points. We will indicate the intervals as follows

$$
\begin{aligned}
& \text { [1] } r \leqq-1, \quad[3]-\frac{1}{2} \leqq r \leqq 0, \quad[5] \quad 1 \leqq r \leqq 2 \text {, } \\
& \text { [2] } r \geqq 2, \quad \text { [4] } 0 \leqq r \leqq 1, \quad \text { [6] }-1 \leqq r \leqq-\frac{1}{2} \text {. }
\end{aligned}
$$

The end points of the intervals are regarded as belonging to both intervals. If $\alpha_{1}$ is in $[a]$ and $\alpha_{2}$ in [b], the corresponding point of $K$ is on a portion which we shall denote by $[a, b]$, where $[a, b]$ and $[b, a]$ are of course identical. In order to have a real point of the surface $[a]$ and $[b]$ must be both even or both odd. We may speak of the portions, therefore, as even and odd portions. The portions $[1,3],[3,5],[5,1],[2,4],[4,6]$, $[6,2]$ each make up one piece. They are the six pieces which are not cut by the cylinder (3). The portions $[3,5]$ and $[4,6]$ make up the two finite pieces. The portions $[1,1],[3,3]$, $[5,5]$ are on one of the pieces cut by the cylinder and the portions $[2,2],[4,4],[6,6]$ are on the other. At each node there is one even portion and one odd portion. With the exception of the node $(0,0,1,0)$ which connects the two pieces cut by the cylinder, no even portion joins with an even portion and no odd portion with an odd portion at a node. 
The following table gives the sixteen nodes and tropes and also the portions joining at each node. The numbers in the first column are the symbols for the nodes (or tropes) according to the scheme given in the discussion of the general surface. The symmetry is again in evidence here. If we interchange $x$ with $z$ and $y$ with $t$, the nodes and tropes are interchanged as follows: $(0)$ with $(1,2,3,4),(i)$ with $(j, k, l)$ and $(i, j)$ with $(k, l)$.

$\begin{array}{cccc} & \text { nodes } & \text { tropes } & \text { portions } \\ (0) & (1,0,0,0) & y=0 & {[2,4][1,3]} \\ (1) & (1,0,1,1) & y-z+t=0 & {[4,4][3,5]} \\ (2) & (1,0,1,-1) & y+z+t=0 & {[4,6][1,3]} \\ (3) & (4,0,1,2) & 4 y-2 z+t=0 & {[2,4][3,5]} \\ (4) & (1,0,4,-2) & y+2 z+4 t=0 & {[4,6][3,3]} \\ (1,2) & (0,2,3,-2) & 2 x-2 z-3 t=0 & {[4,6][5,1]} \\ (2,3) & (1,-2,-1,1) & 2 x+y-z-t=0 & {[6,2][5,1]} \\ (1,3) & (3,2,-3,1) & 2 x-3 y+z+3 t=0 & {[2,4][5,5]} \\ (2,4) & (-3,1,3,2) & x+3 y+2 z-3 t=0 & {[6,6][1,3]} \\ (1,4) & (1,-1,-1,2) & x+y-2 z-t=0 & {[4,6][3,5]} \\ (3,4) & (3,-2,0,2) & 2 x+3 y-2 z=0 & {[6,2][3,5]} \\ (1,2,3) & (4,-2,1,0) & 2 x+4 y+t=0 & {[6,2][1,3]} \\ (2,3,4) & (1,1,1,0) & x-y-t=0 & {[2,4][5,1]} \\ (3,4,1) & (1,-1,1,0) & x+y+t=0 & {[6,2][1,1]} \\ (4,1,2) & (1,2,4,0) & 2 x-y-4 t=0 & {[2,2][5,1]} \\ (1,2,3,4) & (0,0,1,0) & t=0 & {[2,2][4,4][6,6]} \\ & & & {[1,1][3,3][5,5]}\end{array}$

Mississippi Woman's College 